\title{
UMA ANÁLISE INTRASSETORIAL E INTERSETORIAL DO GRAU DE INOVAÇÃO DE EMPRESAS DE PEQUENO PORTE DO ESTADO DE PERNAMBUCO
}

\section{Breno José Burgos Paredes}

Especialista em Administração Financeira pela Universidade de Pernambuco - UFPE brenoparedes@globo.com (Brasil)

\section{Guilherme Alves de Santana}

Mestre em Ciência da Informação pela Universidade Federal de Pernambuco - UFPE guilherme.alves.santana@gmail.com (Brasil)

\section{Thiago Neves Cunha}

Bacharel em Administração pela Universidade Federal de Pernambuco - UFPE nevesc.thiago@gmail.com (Brasil)

\section{Joás Tomaz de Aquino}

Bacharel em Administração pela Universidade Federal de Pernambuco - UFPE joastomaz@outlook.com (Brasil)

\section{RESUMO}

O desenvolvimento da inovação nas empresas tem como alicerce o esforço da continuidade na geração de indicadores de desempenho. Entretanto, medidas e avaliações realizadas em empresas frequentemente são pautadas em objetivos e efeitos imediatistas, impossibilitando o esforço do prosseguimento na geração de parâmetros. Para o processo de melhoria gradual do desempenho da organização, as avaliações do grau de inovação devem ser contínuas. Diante disto, este estudo avalia o grau de inovação (GI), o grau de inovação setorial (GIS) e o característico de inovação setorial (CIS) de empresas de pequeno porte (EPPs) pertencentes aos setores de indústria, comércio e serviços do estado de Pernambuco, sob uma perspectiva de análise intrassetorial contínua e intersetorial. Para atingir este objetivo, houve aplicação do diagnóstico Radar da Inovação em 90 EPPs, em três momentos distintos. Os resultados apontaram que o setor de serviços demonstrou maior evolução do grau de inovação quando comparado ao de comércio e indústria. O setor de comércio apresentou evolução do GI em todas as dimensões, enquanto o setor industrial obteve acréscimos em apenas seis dimensões do radar. Por meio do GIS foi possível identificar as dimensões Relacionamento, Clientes e Marca como indutoras nos três setores analisados.

Palavras-chave: Inovação; Radar da inovação; Micro e pequena empresa; Grau de inovação setorial. 


\section{INTRODUÇÃO}

As organizações usam diversos indicadores para uma melhor compreensão do seu desempenho organizacional e do valor que produzem. Entre esses indicadores, a mensuração da inovação está cada vez mais presente na fundamentação de ações estratégicas, devido ao reconhecimento da inovação como fator crítico para o sucesso empresarial, ao passo que permite às empresas manterem vantagens competitivas (Carayannis \& Provance, 2008). Desta maneira, a avaliação do grau de inovação é um elemento essencial para a compreensão da dinâmica da inovação de empresas, como também dos setores econômicos.

Os principais indicadores de inovação utilizados no Brasil englobam as patentes registradas, publicações científicas e recursos humanos (que exercem funções de cientistas e engenheiros em pesquisas). Ainda sobre as empresas nacionais, há ferramentas e pesquisas voltadas a mensuração do grau de inovação organizacional, entre elas, o diagnóstico do Radar da Inovação, por exemplo, é uma ferramenta que vem sendo aplicada em consultorias de inovação em empreendimentos de pequeno porte no Brasil.

Essa ferramenta permite analisar a evolução do estado de inovação, que pode ser específica (uma ou poucas empresas) ou mais abrangente (envolvendo setores e empresas de segmentos diferentes) (Oliveira et al., 2011; 2014; Oliveira, Cavalcanti, \& Paiva Jr, 2013), podendo ser também intraorganizacional (dentro da organização) e interorganizacional (além das fronteiras da empresa) (Armbruster et al., 2008; Lara \& Guimarães, 2013). Neste sentido, é possível avaliar aspectos inerentes ao grau de inovação organizacional (GI) e ao setorial (GIS) e ao característico de inovação setorial (CIS) tanto em nível local ou nacional.

Neste trabalho, optou-se por avaliar o GI, o GIS e o CIS de empresas de pequeno porte (EPPs) pertencentes aos setores de indústria, comércio e serviços do estado de Pernambuco, sob uma perspectiva de análise intrassetorial contínua e intersetorial. Dessa forma, o presente estudo possui as seguintes perguntas de pesquisa: como evoluiu o GI das pequenas empresas nos três setores da economia pernambucana no período de 2012 a 2014? E quais as dimensões da inovação que potencializam a formação do grau de inovação setorial (GIS) e apresentam melhor desempenho e maior potencial de crescimento no período considerado?

Revista de Administração e Inovação, São Paulo, v. 12, n.4 p. 140-161, out./dez. 2015. 


\section{REFERENCIAL TEÓRICO}

\subsection{Inovação}

As pesquisas realizadas para a criação de inovações sempre foram importantes para explicar o comportamento e o desenvolvimento dos mercados (Julien, 2010). Tidd, Bessant e Pavitt (2008) argumentam que a literatura sobre inovação atrela parte do sucesso econômico das organizações à implantação de medidas inovadoras em seus produtos e processos. No sentido econômico, a inovação é completa quando ocorre uma transação comercial envolvendo uma invenção e assim gerando riqueza (Schumpeter, 1988).

A definição de inovação no âmbito empresarial não é simples, podendo ser encontradas explicações distintas, porém de certa forma não são totalmente excludentes (Andreassi, 2007). Uma definição genérica pode ser sugerida pela seguinte equação: inovação = ideia + implementação + resultado, de modo que uma ideia só se transforma em inovação se for implementada com sucesso no mercado (Moreira \& Queiroz, 2007). Outra definição pode ser encontrada na Lei de Inovação, Lei ${ }^{\circ}$ 10.973/2004, que associa à inovação a "introdução de novidade ou aperfeiçoamento no ambiente produtivo ou social que resulte em novos produtos, processos ou serviços” (Brasil, 2004).

Além da multiplicidade de conceitos e definições, a inovação pode ser caracterizada por tipologias variadas. Em estudo publicado no Manual de Oslo (OCDE, 2006), as inovações podem ser enquadradas como de produto (bem ou serviço), de processo, de marketing ou organizacional. Já Andreassi (2007), destaca os cinco tipos de inovações mencionados por Schumpeter (1988): introdução de um novo produto ou mudança qualitativa em produto existente; introdução de um novo método de produção; abertura de um novo mercado; desenvolvimento ou conquista de novas fontes de suprimento de matéria-prima ou outros insumos; mudanças na estrutura da organização. No entanto, há quem classifique as inovações de acordo com seu impacto no mercado, podendo ser do tipo radical e incremental.

Para este estudo, utiliza-se a abordagem da inovação organizacional que está relacionada às "modificações radicais ou incrementais nos processos de práticas de negócios na empresa, interna ou externamente, visando à melhoria no fluxo das atividades organizacionais e posicionamento de mercado" (Silva, 2013, p. 72). Ainda sobre a inovação organizacional, vale ressaltar que pode ser concebida nas dimensões intraorganizacional (dentro da organização) e interorganizacional (relacionadas às novas estruturas ou procedimentos para além das fronteiras da empresa, como relacionamentos com clientes e fornecedores) (Armbruster et al., 2008; Lara \& Guimarães, 2013).

Revista de Administração e Inovação, São Paulo, v. 12, n.4 p. 140-161, out./dez. 2015. 
A implantação de medidas inovadoras nas empresas pode ser compreendida como um processo de aprendizado interativo baseado na tensão entre os indivíduos e a organização, sendo, na maioria das vezes, mais ou menos organizado nas micro e pequenas empresas (MPEs), pois uma pequena mudança gera outras, criando um turbilhonar, no qual surgem novos ciclos de mudanças, conforme ilustrado a seguir:

\section{Figura 1 - Funcionamento espiral e turbilhonar da inovação em um bom número de MPEs}

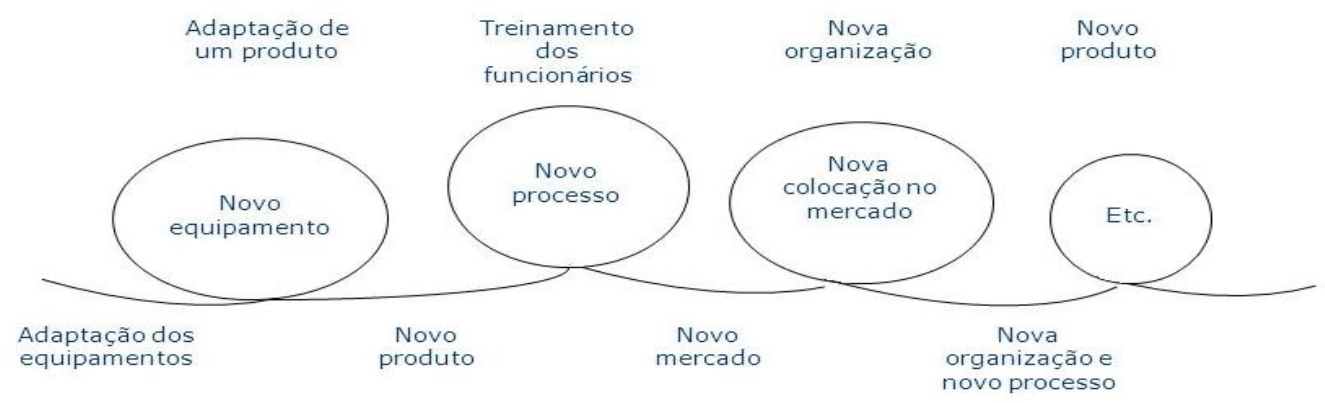

Fonte: Julien, (2010).

A inovação também pode ser um indicador utilizado por empreendedores para explorar a mudança como uma oportunidade para a diferenciação de um negócio ou um serviço. Em quase todas as indústrias o funcionamento das organizações está cada vez mais condicionado a sua capacidade de produzir inovações (Ukko \& Saunila, 2013). Assim, estes devem buscar fontes que indiquem o surgimento de uma nova oportunidade para que uma inovação tenha êxito (Drucker, 2002).

O desenvolvimento da inovação nas empresas tem como alicerce o esforço da continuidade na geração de indicadores de desempenho. Entretanto, medidas e avaliações realizadas frequentemente são pautadas em objetivos e efeitos imediatistas, impossibilitando o esforço de continuidade na geração de parâmetros. Para o processo de melhoria gradual do desempenho da organização, as avaliações do grau de inovação devem ser contínuas.

Sawhney et al. (2006) argumentava que as organizações que possuem uma visão limitada da inovação podem perder oportunidades de crescimento, enquanto a identificação e controle de uma maior número de dimensões da inovação pode proporcionar sinergia para provocar mudanças diante das intempéries de mercado. Buscando o mapeamento dessas dimensões, os autores anteriormente citados propõem o chamado radar da inovação que desdobra a inovação em quatro diferentes dimensões, a saber: (i) o que é oferecido pela empresa; (ii) quais os clientes por eles servidos; (iii) os processos que a empresa utiliza para atender as solicitações dos clientes; e (iv) os aspectos do mercado 
que interferem no que oferecido pela empresa aos clientes. A partir dessas quatros dimensões âncoras, existe o desdobramento de mais nove dimensões que compõe o chamado radar da inovação.

O Radar da Inovação, por exemplo, permite uma avaliação contínua do grau de inovação. Este diagnóstico é baseado em estudos de Sawhney, Wolcoot e Arroniz (2006) e envolve treze dimensões: Oferta, Plataforma, Marca, Clientes, Soluções, Relacionamento, Agregação de valor, Processos, Organização, Cadeia de fornecimento, Presença, Rede e Ambiência inovadora.

\subsection{Estudos de mensuração da inovação pelo radar da inovação}

Conforme visto na seção anterior, uma ferramenta que vem sendo utilizada para mensurar o grau de inovação em pequenas empresas é o Diagnóstico Radar da Inovação, já que abarca mais dimensões que outros modelos de mensuração, possibilitando uma avaliação em um sentido mais amplo, segundo Oliveira et al. (2011). Dependendo da perspectiva de análise, o referido diagnóstico pode permitir uma análise geral do grau inovativo de empresas, como também específico das suas dimensões.

Sobre pesquisa específica sobre suas dimensões, Santana, Paredes e Valente (2013) verificaram que as principais inovações na dimensão relacionamento ocorriam através de ações em redes sociais, e especificamente, por meio de promoções, comunicação e divulgação dos produtos e serviços de mídias como o facebook, twitter, skype e foursquare. Em estudo semelhante, mas na dimensão marca, Paredes, Valente e Santana (2013) identificaram que empresas do segmento metal mecânico estavam em nível inferior a uma organização ocasionalmente inovadora, consequência da falta de planejamento de marketing e ausência da preocupação por parte dos empresários em proteger a marca da sua empresa.

Na medida em que o Radar da Inovação possibilita uma avaliação de dimensões específicas, também propicia análises setoriais ou de uma única empresa, possibilitando uma análise abrangente ou uma análise estritamente específica. Sobre o primeiro tipo de análise, Rocha et al (2011) avaliaram MPEs do setor de fabricação de móveis do estado de Pernambuco, e averiguaram semelhanças entre o comportamento do grau organizacional e o grau de inovação, confirmando a hipótese de que para MPEs existe um nível mínimo de estrutura organizacional capaz de estimular a inovação no ambiente interno e que a estrutura da organização também depende do ambiente externo. Silva Néto e Teixeira (2011) possibilitaram uma comparação entre o grau de inovação de MPEs da cadeia têxtil-confecção da capital e do inteiro do estado de Sergipe. Os autores concluíram que os resultados obtidos pelas empresas instaladas na capital Aracaju foram, de modo geral, superiores aos obtidos pelas empresas do interior do estado.

Revista de Administração e Inovação, São Paulo, v. 12, n.4 p. 140-161, out./dez. 2015. 
Sobre pesquisas do grau inovativo de empresas, ressalta-se o trabalho de Paredes, Santana e Fell (2014) na análise do grau de inovação organizacional em uma EPP do setor metal mecânico pernambucano que identificou que a evolução inovativa da empresa foi resultante de ações voltadas ao desenvolvimento de logotipo e constituição de uma identidade visual, identificação dos principais gargalos na gestão da empresa, criação de novos canais de comunicação com o cliente e elaboração de estratégias para aperfeiçoar e padronizar os processos administrativos.

Entretanto, Oliveira et al. (2011) apontam que o radar tem uma limitação que é não abordar as empresas levando em consideração a heterogeneidade dos setores a que elas pertencem. Por este motivo, os autores supracitados propõem um novo modelo de mensuração da inovação, chamado grau de inovação setorial (GIS) e característico de inovação setorial (CIS), que permitem avaliar o grau de inovação de empresas referenciadas com o seu setor de atuação. No GIS e no CIS, o resultado da inovação obtido em cada empresa permite compará-las em um ou entre setores, demonstrando se "há possibilidade de uma empresa mediana em termos de inovação, mas situada num setor propenso à inovação, ser considerada mais inovadora que a empresa mais inovadora de um setor com dificuldades naturais para inovar" (Oliveira et al., 2011, p. 13). Utilizando esses métodos, Oliveira, Cavalcanti e Paiva Jr. (2013) e Oliveira et al. (2014) puderam realizar uma análise comparativa entre oito segmentos diferentes, possibilitando avaliar qual o mais inovador e qual era mais embrionário.

Neste sentido, os estudos citados sobre mensuração da inovação por meio da utilização do Radar da Inovação corroboram a consistência da aplicação da ferramenta para aferir o estado inovativo de uma empresa. Por outro lado, o GIS e o CIS consolida tal tipo de mapeamento, pois demonstram os impactos provenientes da inovação por outras vertentes ainda mais confiáveis.

\section{PROCEDIMENTOS METODOLÓGICOS}

A presente pesquisa se caracteriza por ser predominantemente quantitativa, pois busca a mensuração do grau de inovação de determinados setores econômicos e a identificação das dimensões relevantes para cada setor estudado. Para a realização deste estudo, optou-se pela utilização do método de levantamento, pois possibilita a "descrição quantitativa ou numérica de tendências, atitudes ou opiniões de uma população, estudando-se uma amostra desta população" (Creswell, 2010, p. 178). Quanto aos fins, a pesquisa é exploratória. Quanto aos meios, este estudo foi construído a partir de uma pesquisa bibliográfica e uma pesquisa de campo.

Revista de Administração e Inovação, São Paulo, v. 12, n.4 p. 140-161, out./dez. 2015. 
A amostra da pesquisa realizada em campo foi de 90 EPPs, pertencentes aos setores da indústria, comércio e serviços, situadas na Região Metropolitana do Recife (RMR), no estado de Pernambuco. A distribuição das empresas entre os setores ocorreu de forma igualitária, ou seja, cada setor foi constituído por um grupo de 30 empresas, da seguinte forma: setor industrial - formado por EPPs dos segmentos de plástico, metal mecânico, gráfico, químico e de refrigeração; setor comercial composto pelos segmentos de bares e restaurantes, padarias e de comércio varejista em geral; setor de serviços - integrado por empresas como agências de turismo, locadoras de veículos, empresas organizadoras de eventos e casas de recepção. A distribuição das empresas entre os setores foi realizada pela atividade principal dos empreendimentos, com base no Código Nacional de Atividade Econômica (CNAE).

O instrumento utilizado para mensurar o grau de inovação organizacional das referidas empresas foi o diagnóstico Radar da Inovação. O diagnóstico é composto por 12 dimensões propostas por Sawhney e Chen (2010) com o acréscimo da dimensão 'ambiência inovadora', sugerido por Bachmann e Destefani (2008), conforme o Quadro 1.

Quadro 1 - Conceituação e detalhamento das dimensões do Radar da Inovação

\begin{tabular}{|c|c|c|}
\hline $\mathbf{N}^{\mathbf{o}}$ & DIMENSÕES & CONCEITO \\
\hline 1 & Oferta & Produtos oferecidos pela empresa ao mercado. \\
\hline 2 & Plataforma & $\begin{array}{l}\text { Conjunto de componentes comuns, métodos de montagem ou tecnologias } \\
\text { utilizados, analisando o modo de fazer e de prestar serviços. }\end{array}$ \\
\hline 3 & Marca & Forma como a empresa transmite sua imagem ao cliente. \\
\hline 4 & Clientes & $\begin{array}{l}\text { Pessoas ou organizações que usam serviços ou consomem produtos, } \\
\text { satisfazendo às suas necessidades. }\end{array}$ \\
\hline 5 & Soluções & $\begin{array}{l}\text { Combinação customizada de bens, serviços e informações capazes de } \\
\text { solucionar o problema do cliente. }\end{array}$ \\
\hline 6 & Relacionamento & $\begin{array}{l}\text { Experiência do cliente, representando tudo o que o consumidor vê, ouve, } \\
\text { sente ou experimenta quando interage com a empresa. }\end{array}$ \\
\hline 7 & $\begin{array}{l}\text { Agregação de } \\
\text { Valor }\end{array}$ & $\begin{array}{l}\text { Forma pela qual a empresa percebe novas formas de captar e transferir valor } \\
\text { ao cliente. }\end{array}$ \\
\hline 8 & Processos & $\begin{array}{l}\text { Configurações das atividades usadas na condução das operações internas da } \\
\text { empresa, a fim de produzir um produto ou prestar um serviço. }\end{array}$ \\
\hline 9 & Organização & $\begin{array}{l}\text { Modo como a empresa está estruturada, relacionando as parcerias } \\
\text { estabelecidas com o papel e a responsabilidade dos colaboradores. }\end{array}$ \\
\hline 10 & $\begin{array}{l}\text { Cadeia de } \\
\text { Fornecimento }\end{array}$ & $\begin{array}{l}\text { Sequência de atividades que movem produtos, serviços e informações da } \\
\text { origem à entrega, abrangendo aspectos relacionados com a logística do } \\
\text { negócio. }\end{array}$ \\
\hline 11 & Presença & $\begin{array}{l}\text { Canais de distribuição que a empresa utiliza para colocar seus produtos no } \\
\text { mercado, além dos locais onde esses itens podem ser adquiridos. }\end{array}$ \\
\hline 12 & Rede & $\begin{array}{l}\text { Aspectos relacionados com a rede que conecta a empresa e seus produtos } \\
\text { aos clientes, fazendo com que os recursos utilizados tragam uma } \\
\text { comunicação ágil e eficaz entre empresa e cliente. }\end{array}$ \\
\hline 13 & $\begin{array}{l}\text { Ambiência } \\
\text { Inovadora }\end{array}$ & $\begin{array}{l}\text { Conjunto de ferramentas, processos e atitudes que promovem ou motivam } \\
\text { os empregados a criar algo novo ou melhorias. }\end{array}$ \\
\hline
\end{tabular}

Fonte: Oliveira et al (2014). 
Como visto, o diagnóstico é constituído por 13 dimensões. Cada uma destas dimensões possui variáveis que visam avaliar o nível de ocorrência de inovações nos últimos três anos de existência da empresa. Para cada variável são atribuídas pontuações que podem variar entre 1 e 5, sendo atribuído valor 1 para uma dimensão pouco inovadora, 3 para as dimensões inovadora ocasional e e 5 para a inovação sistêmica.

O radar foi aplicado três vezes em cada um dos 90 empreendimentos, entre os anos de 2012 [buscando avaliar o grau de inovação inicial (R0)], 2013 [a evolução do nível de inovação após a execução de ações sugeridas (R1)] e 2014 (terceiro momento (R2)]. Para cada momento de aplicação do diagnóstico, ocorreram as seguintes etapas: entrevista e aplicação do diagnóstico, recebimento e tratamento dos dados, em planilha eletrônica do Microsoft Excel; cálculo do grau de inovação (GI) da amostra; análise dos extratos e comparações, e; consolidação do material.

Após a coleta dos dados, foram obtidas as médias, os escores máximos e mínimos de cada dimensão por setor nos três períodos de realização do diagnóstico (R0, R1 e R2). Em seguida, foram realizadas duas análises: a análise intrassetorial que se constituiu no exame dos acréscimos no grau de inovação (GI) médio dos setores, ocorridos entre o R0 e R2, e; posteriormente a análise intersetorial para o momento R2, baseada no cálculo do grau de inovação setorial (GIS) e no característico de inovação setorial (CIS) desenvolvido por Oliveira et al. (2011a, 2011b, 2014) e Oliveira, Cavalcanti e Paiva Jr. (2013), uma vez que permitem uma análise mais analítica e crítica ao levar em consideração os elementos chaves que caracterizam as especificidades da dinâmica de um setor.

Segundo Oliveira et al. (2014), a necessidade de utilização do GIS e do CIS nasce da dificuldade do diagnóstico radar de inovação em medir a inovação global e a heterogeneidade de cada setor. Ou seja, um lançamento de um novo produto para a indústria metal mecânica tem um impacto superior ao de um lançamento de um novo produto no segmento de bares e restaurantes, a depender do mix de produtos. Essas peculiaridades incrementam um diferencial na estruturação do GIS.

Diante da constatação da heterogeneidade dos setores, é proposto o grau de inovação setorial por empresa (GIS), que é definido pela Equação 1, a seguir:

$$
G I S=\sum_{k=1}^{13} p_{k} D_{M k}
$$

Onde:

I. $\quad \mathrm{D}_{M k}$ é o valor da dimensão da inovação $k$ para o setor $M$;

II. $\quad p_{k}$ representa o peso das dimensões da inovação. 
Por sua vez, o peso das dimensões da inovação $p_{k}$ é obtido através do seguinte modelo de otimização:

Função Objetivo: $\quad \operatorname{Max} \sum_{k=1}^{13} p_{k} D_{M k}$

Sujeito a: $\quad \sum_{k=1}^{13} p_{k}=1$

$$
\begin{array}{ccc}
p_{k} \geq 0,05 & \text { para } & \forall k \\
D_{M k} p_{k} \leq 0,5 & \text { para } & \forall k
\end{array}
$$

Analisando a programação linear acima, a função objetivo tem como propósito maximizar a média ponderada, composta dos pesos e os valores de cada dimensão de um setor. Como restrições têm-se: a soma dos pesos deve ser igual a 1; cada peso deve ser maior ou igual a 0,05 (esse valor foi considerado para que nenhuma dimensão obtivesse valor zero na ponderação, ou seja, o valor de 0,05 representa o mínimo que uma dimensão pode ter). Por fim, a ponderação de cada dimensão de inovação $\left(\mathrm{D}_{M k} \cdot \mathrm{p}_{k}\right)$ deve ser menor ou igual a 0,5 para evitar que apenas uma dimensão se potencialize e iniba a ação das outras.

O característico de inovação setorial (CIS), por sua vez, permite destacar as dimensões que apresentaram maior desempenho para determinado setor, por meio do uso das funções da atribuição das características da inovação setorial, caracterizando-se como uma ferramenta complementar ao GIS. Essa métrica tem como objetivo destacar as distâncias entre o valor médio de uma dimensão que teve o melhor desempenho em determinado setor e os valores obtidos por cada dimensão desse mesmo setor. Ou seja, o CIS nos permite mensurar a distância entre os valores médios das dimensões em comparação ao benchmarking interno do setor. O CIS pode ser alcançado por meio da Equação 3:

$$
\text { Medd }_{k s}=\left(1-\frac{\text { Maxmedd }_{s}-d_{i k}}{\text { Maxmedd }_{s}}\right) \times 5
$$

Onde:

I. $\quad M e d d_{k s}$ é o valor médio ajustado da dimensão de inovação $k$ para o setor $s$, e indica quão próximo ao benchmark o setor se encontra em alguma dimensão;

II. Maxmedd é o valor máximo dos valores médios das $k$ dimensão da inovação para as $i$ empresas do setor $s$; e,

III. $\quad d_{i k}$ é o valor médio da dimensão da inovação $k$ do setor $\mathrm{s}$.

O CIS médio setorial (em forma de vetores) das 13 dimensões caracteriza-se pela seguinte Equação 4:

Revista de Administração e Inovação, São Paulo, v. 12, n.4 p. 140-161, out./dez. 2015. 


$$
\operatorname{MedCIS}_{s}=\left(\operatorname{Medd}_{1, s}, \ldots, \operatorname{Medd}_{13, s}\right)
$$

Para complementar a análise, faz-se necessário ainda identificar também o quanto cada dimensão deve se desenvolver para alcançar o seu valor máximo. Para identificar este potencial de acréscimo utiliza-se a métrica 5 a seguir:

$$
\operatorname{Maxd}_{k s}=\left(\frac{\operatorname{Maxd}_{i s}-d_{i k}}{\operatorname{Maxd}_{i s}}\right) \times 5
$$

Onde:

I. $\quad \operatorname{Maxd}_{k s}$ é o valor máximo ajustado das $k$ dimensões de inovação para o setor $s$; indicando o quanto esta dimensão pode evoluir em relação ao concorrente do mesmo setor. Quanto maior o valor deste indicador, maior o potencial de crescimento da dimensão analisada;

II. $\quad \operatorname{Maxd}_{i k}$ é o valor máximo dos valores das dimensões da inovação $k$ para as $i$ empresas do setor s;

III. $d_{i k}$ é o valor médio da dimensão da inovação $k$ do setor $s$.

Já o CIS setorial para as 13 dimensões analisadas pelo radar (também descrito na forma vetorial) é definido como sendo:

$$
\operatorname{MaxCIS}_{s}=\left(\operatorname{Maxd}_{1, s}, \ldots, \operatorname{Maxd}_{13, s}\right)
$$

Apesar da inovação, no ambiente organizacional, ser caracterizada pelo processo de mudança permanente, a aplicação do GIS e do CIS no momento R2 possibilitou a captura das dinâmicas e incrementos realizados de 2012 até 2014. Dessa forma, tem-se uma representação de um diagnóstico preciso das dimensões no momento considerado e a composição de um grau de inovação que represente fidedignamente a realidade do setor.

\section{ANÁLISE E DISCUSSÃO DOS RESULTADOS}

\subsection{Análise intrassetorial}

Revista de Administração e Inovação, São Paulo, v. 12, n.4 p. 140-161, out./dez. 2015. 
A seguir serão discutidos os resultados relativos as 90 empresas dos setores da indústria, comércio e serviços. Portanto, serão apresentadas as médias dos GIs das empresas por setor nos momentos R0, R1 e R2 (Tabela1), após a implementação de ações inovadoras.

Tabela 1 - Médias nos momentos R0, R1 e R2 da amostra de 90 empresas

\begin{tabular}{|c|c|c|c|c|c|c|c|c|c|}
\hline \multirow[t]{2}{*}{ DIMENSÕES } & \multicolumn{3}{|c|}{ INDÚSTRIA } & \multicolumn{3}{|c|}{ SERVIÇOS } & \multicolumn{3}{|c|}{ COMÉRCIO } \\
\hline & R0 & R1 & $\mathbf{R 2}$ & R0 & R1 & $\mathbf{R 2}$ & $\mathbf{R 0}$ & R1 & $\mathbf{R 2}$ \\
\hline OFERTA & 2,000 & 2,027 & 2,147 & 2,250 & 2,600 & 2,767 & 3,487 & 3,62 & 3,707 \\
\hline PLATAFORMA & 4,467 & 4,500 & 4,500 & 2,733 & 3,367 & 3,733 & 4,300 & 4,43 & 4,767 \\
\hline MARCA & 3,100 & 3,400 & 3,733 & 3,533 & 3,900 & 4,000 & 3,533 & 3,86 & 3,933 \\
\hline CLIENTES & 2,204 & 2,271 & 2,341 & 2,713 & 3,320 & 3,747 & 2,330 & 2,70 & 3,117 \\
\hline SOLUÇÕES & 1,833 & 1,867 & 1,967 & 3,033 & 3,433 & 3,900 & 1,800 & 2,16 & 2,567 \\
\hline RELACIONAMENTO & 2,467 & 2,900 & 3,300 & 3,433 & 3,800 & 3,967 & 2,433 & 3,00 & 3,367 \\
\hline AGR. VALOR & 1,400 & 1,400 & 1,600 & 1,467 & 1,900 & 2,133 & 1,600 & 2,00 & 2,333 \\
\hline PROCESSOS & 1,859 & 2,073 & 2,226 & 1,850 & 2,210 & 2,620 & 1,697 & 1,88 & 2,120 \\
\hline ORGANIZAÇÃO & 1,750 & 1,967 & 2,217 & 2,783 & 3,183 & 3,583 & 1,833 & $\underset{7}{2,36}$ & 2,900 \\
\hline $\begin{array}{c}\text { CAD. } \\
\text { FORNECIMENTO }\end{array}$ & 1,933 & 2,000 & 2,200 & 1,933 & 2,133 & 2,333 & 1,933 & $\begin{array}{c}2,26 \\
7\end{array}$ & 2,533 \\
\hline PRESENÇA & 1,633 & 1,667 & 1,700 & 1,567 & 1,767 & 2,067 & 1,467 & $\underset{7}{1,56}$ & 1,600 \\
\hline REDE & 2,200 & 2,933 & 3,333 & 1,867 & 3,400 & 4,533 & 1,667 & $\begin{array}{c}1,80 \\
n\end{array}$ & 2,333 \\
\hline AMB. INOVADORA & 1,623 & 1,850 & 1,880 & 1,860 & 2,160 & 2,483 & 1,827 & $\begin{array}{c}2,09 \\
0\end{array}$ & 2,403 \\
\hline $\begin{array}{c}\text { GRAU DE } \\
\text { INOVAÇÃO }\end{array}$ & 2,150 & 2,336 & 2,502 & 2,349 & 2,810 & 3,168 & 2,267 & $\begin{array}{c}2,56 \\
2\end{array}$ & 2,863 \\
\hline
\end{tabular}

Fonte: Dados da Pesquisa de Campo (2015).

Observa-se que houve uma evolução no grau de inovação médio (GI) de todos os setores no decorrer da pesquisa, entre os momentos R0, R1 e R2. Destaca-se que os acréscimos dos escores são oriundos da implantação de ações inovadoras pelas empresas estudadas (que visavam aprimorar os processos, produtos, atividades mercadológicas e/ou organizacionais). Conforme observado por Oliveira et al (2011a, 2011b), os escores não diminuem, uma vez que a organização continua realizando ações inovadoras de forma permanente e direcionada.

Apesar da Tabela 1 possibilitar uma visualização do GI médio das empresas, é necessário observar o desempenho individual de cada dimensão da inovação. Para isto, o Gráfico 1 apresenta uma comparação intrassetorial dos setores industrial, comercial e de serviços, entre os momentos R0, R1 e R2, evidenciando a evolução das dimensões inovadoras. 


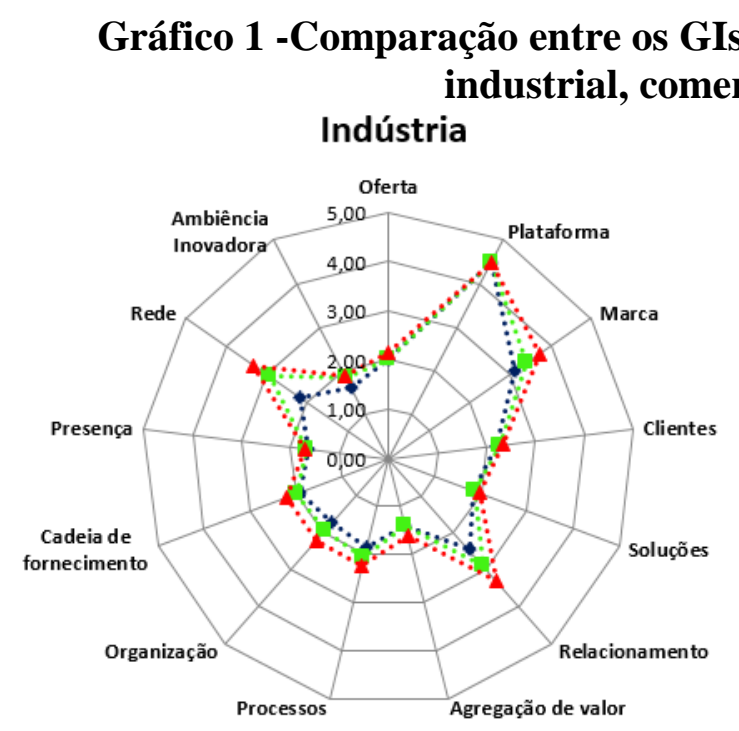

\section{e de serviços}

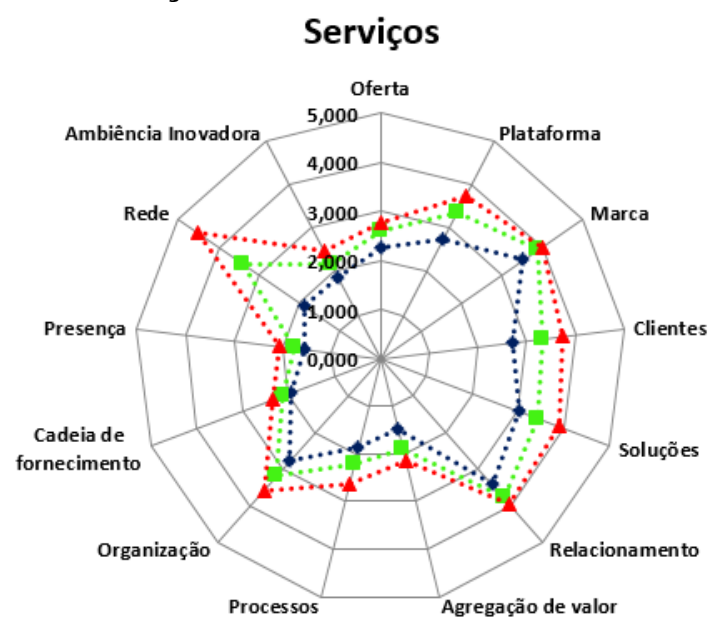

\section{Comércio}

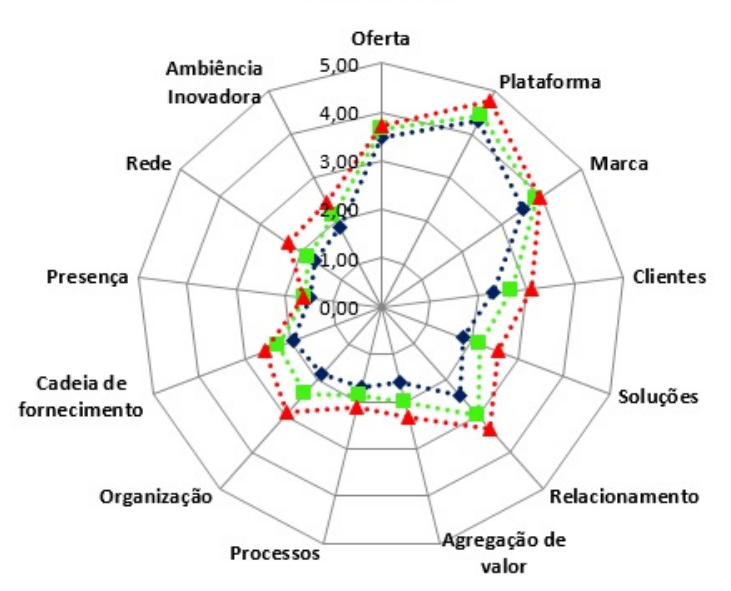

Legenda

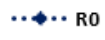

……R1

$\cdots \mathbf{R} 2$

Fonte: Dados da Pesquisa de Campo (2015).

As dimensões que obtiveram melhor desempenho no setor industrial foram: Marca, Relacionamento, Organização e Rede; obtendo um acréscimo superior a $20 \%$ cada, entre os diagnósticos R0 e R2. Observa-se que este resultado se assemelha ao identificado por Paredes, Valente e Santana (2013), onde identificaram incremento na dimensão marca e por Rocha et al (2011) em estudo sobre a indústria moveleira. A dimensão Plataforma se destacou positivamente por alcançar o maior valor $(4,50)$ entre as treze dimensões do setor industrial. Por sua vez, o setor serviços apresentou visíveis avanços em todas as suas dimensões do radar, no comparativo R0-R1, e R1-R2. Das 13 dimensões do radar, 9 apresentaram uma evolução acima dos 28,5\% entre os radares R0 e R2. Dentre estas, a dimensão Rede destacou-se positivamente por obter um expressivo acréscimo de 142\%, entre o primeiro e último radar.

$\mathrm{Na}$ avaliação intrassetorial do comércio, apenas as dimensões Oferta, Plataforma, Marca e Presença não sofreram acréscimos significativos, apresentando variações entre o R0 e R2 inferiores a 
12\%. Em contrapartida, a dimensão Organização obteve acréscimo de 58\% na comparação entre os radares.

Em síntese, ressalva-se que o setor industrial obteve o menor desempenho, em termos de evolução do GI, frente aos demais setores. Porém, destaca-se que a indústria demonstrou o aprimoramento das dimensões que estão diretamente associadas ao contato direto com os clientes (Marca, Relacionamento e Rede), evidenciando uma crescente preocupação com a imagem pública da empresa e com a forma que ele se comunica com a sua clientela. Por outro lado, conforme Ukko e Saunila (2013), o setor de serviços obteve a maior evolução do GI, tal avanço deve-se a característica do próprio setor que se destaca por buscar novos conhecimentos externos a empresa (tais como consultorias, apoio de entidades voltadas ao suporte empresarial) e possui maior flexibilidade para promover soluções inovadoras em seus empreendimentos

Já o setor de comércio obteve avanços significativos nas dimensões que possuem papéis fundamentais na atividade, uma vez que estão diretamente relacionadas à capacidade de ofertar novos produtos dentro da limitação física e material, proposição de novas soluções que gerem receita extra, ao relacionamento entre empresa e cliente, e as atividades ligadas à logística e estoque.

A análise do GI tradicional permitiu visualizar que os três setores apresentam indicadores semelhantes no radar R0, uma vez que Indústria, Serviços e Comércio apresentaram médias de 2,15, 2,35 e 2,27, respectivamente. Todavia, no momento R2 o setor de serviços demonstrou maior evolução, obtendo indicador de 3,17, enquanto, os setores de indústria e comércio apresentaram GI de 2,50 e 2,86 respectivamente.

Em suma, o setor de serviços exibiu um avanço de $35 \%$, entre os períodos R0-R2, sendo o maior, seguido pelos setores de comércio $(26,3 \%)$ e indústria (16,4\%). Especificamente, a dimensão Plataforma se destacou nos setores de indústria e comércio, enquanto, Rede obteve destaque no setor de serviços. Neste contexto, os indicadores de inovação gerados por meio da metodologia radar da inovação possibilitou o mapeamento do cenário inovativo dos setores alvo da observação deste estudo.

\subsection{Análise intersetorial: o diagnóstico realizado pelo GIS e CIS}

Ao contrário das premissas do GI tradicional, onde cada dimensão possui o mesmo peso, com exceção da dimensão ambiência inovadora (peso 0,2), o GIS busca identificar às dimensões relevantes para o setor, dada a heterogeneidade setorial. Na Tabela 2 estão descritos os pesos encontrados para

Revista de Administração e Inovação, São Paulo, v. 12, n.4 p. 140-161, out./dez. 2015. 
cada dimensão nos três setores estudados, durante o momento R2 da pesquisa, utilizando o modelo otimização descrito na Equação 2.

Tabela 2 - Pesos por setores e GIS no momento R2

\begin{tabular}{c|c|c|c}
\hline \multirow{2}{*}{ DIMENSÕES } & \multicolumn{3}{|c}{ Pesos por setores econômicos } \\
\cline { 2 - 4 } & INDÚSTRIA & COMÉRCIO & SERVIÇOS \\
\hline OFERTA & 0,050 & 0,135 & 0,050 \\
\hline PLATAFORMA & 0,111 & 0,105 & 0,050 \\
\hline MARCA & 0,134 & 0,127 & 0,125 \\
\hline CLIENTES & 0,053 & 0,085 & 0,110 \\
\hline SOLUÇÕES & 0,050 & 0,050 & 0,128 \\
\hline RELACIONAMENTO & 0,152 & 0,149 & 0,126 \\
\hline AGREGAÇÃO DE & 0,050 & 0,050 & 0,050 \\
\hline VALOR & 0,050 & 0,050 & 0,050 \\
\hline PROCESSOS & 0,050 & 0,050 & 0,050 \\
\hline ORGANIZAÇÃO & 0,050 & 0,050 & 0,050 \\
\hline FORNECIMENTO & 0,050 & 0,050 & 0,050 \\
\hline PRESENÇA & 0,150 & 0,050 & 0,110 \\
\hline REDE & 0,050 & 0,050 & 0,050 \\
\hline AMBIÊNCIA & $\mathbf{2 , 9 2 2}$ & $\mathbf{3 , 2 0 3}$ & $\mathbf{3 , 5 0 0}$ \\
\hline INOVADORA & GIS & Pand
\end{tabular}

Fonte: Dados da Pesquisa de Campo (2015).

Como se percebe no setor industrial, as dimensões que possuem um impacto relevante no grau de inovação setorial por ordem decrescente são: Relacionamento $(0,152)$, Rede $(0,150)$; Marca $(0,134)$ e Plataforma $(0,111)$ - sendo está última também destaque do setor industrial nos resultados das pesquisas de Oliveira et al (2014) e Oliveira, Cavalcanti e Paiva Jr. (2013). Essas dimensões estão em consonância com a atividade fim do setor que é marcada pela estruturação de um processo produtivo e elos à montante e à jusante na cadeira produtiva, na busca de transmitir valores e experiências ao cliente por meio do produto oferecido.

No setor de comércio as dimensões relevantes identificadas foram: Relacionamento $(0,149)$; Oferta (0,135); Marca $(0,127)$, Plataforma $(0,105)$ e Clientes $(0,085)$. O referido resultado deriva-se da oferta de produtos inovadores, possuir uma marca forte, valorização das experiências com o cliente e, sobretudo, estabelecimento de vínculos duradouros com os mesmos. Estas ações fazem com que a empresa esteja propensa a inovar quando valoriza as dimensões citadas.

Por conseguinte, o setor de serviços apresentou as seguintes dimensões críticas para inovação organizacional: Soluções $(0,128)$; Relacionamento $(0,126)$; Marca $(0,125)$; Rede e Clientes $(0,110)$. Como se percebe, as dimensões Soluções, Relacionamento e Marca estão próximas nesse setor, revelando que como manter um bom relacionamento com o cliente, buscando atender as suas 
necessidades de forma customizada e transmitir valores almejados é importante, e um recurso de diferenciação para as empresas do setor.

Nos três setores de atuação, os dados revelam também que entre as 90 empresas da amostra selecionada para as EPPs pernambucanas, as dimensões Relacionamento, Marca e Clientes estão sempre presentes como essenciais na constituição do GIS, com pesos diferentes. Este resultado, de acordo com Oliveira et al (2011a), comprovam que o empresariado pernambucano opta por inovar nessas dimensões, por gerar um impacto direto na relação com o cliente.

Por fim, a Tabela 2 ainda fornece os indicadores dos GISs. Comparando-os com os valores encontrados pelo GI na Tabela 1 percebem-se claras discrepâncias entre os valores. No entanto, o setor de serviços demonstrou-se mais inovador nas duas medidas utilizadas (GI e GIS), seguido do comércio e da indústria, o que havia sido identificado também por Oliveira et al. (2011).

Após a realização desse mapeamento inicial, a Tabela 3 descreve os valores do MedCIS $\mathrm{S}$ e MáxCIS de forma a complementar a análise iniciada pelo GIS.

Tabela 3 - Característicos do setor industrial no momento R2

\begin{tabular}{c|c|c|c|c|c}
\hline DIMENSÕES & Média & Máximo & Mínimo & MedCIS $_{\mathbf{S}}$ & MáxCIS $_{\mathbf{S}}$ \\
\hline OFERTA & 2,147 & 4,200 & 1,000 & 2,385 & 2,444 \\
\hline PLATAFORMA & 4,500 & 5,000 & 3,000 & 5,000 & 0,500 \\
\hline MARCA & 3,733 & 5,000 & 2,000 & 4,148 & 1,267 \\
\hline CLIENTES & 2,341 & 5,000 & 1,000 & 2,601 & 2,659 \\
\hline SOLUÇÕES & 1,967 & 4,000 & 1,000 & 2,185 & 2,542 \\
\hline RELACIONAMENTO & 3,300 & 5,000 & 1,000 & 3,667 & 1,700 \\
\hline AGREGAÇÃO DE VALOR & 1,600 & 3,000 & 1,000 & 1,778 & 2,333 \\
\hline PROCESSOS & 2,226 & 3,000 & 1,300 & 2,473 & 1,291 \\
\hline ORGANIZAÇÃO & 2,217 & 3,500 & 1,000 & 2,463 & 1,833 \\
\hline CAD. FORNECIMENTO & 2,200 & 5,000 & 1,000 & 2,444 & 2,800 \\
\hline PRESENÇA & 1,700 & 3,000 & 1,000 & 1,889 & 2,167 \\
\hline REDE & 3,333 & 5,000 & 1,000 & 3,704 & 1,667 \\
\hline AMBIÊNCIA INOVADORA & 1,880 & 2,500 & 1,300 & 2,089 & 1,240 \\
\hline
\end{tabular}

Fonte: Dados da Pesquisa de Campo (2015).

Utilizando como ponte de corte o valor numérico de 3 para analisar o MedCISs [por representar um valor onde a inovação para o setor é tida como ocasional (média)], esse indicador revela que as dimensões Plataforma (benchmark interno), Marca, Relacionamento e Rede são as que apresentaram o melhor desempenho entre as demais dimensões para a indústria. Vale ressaltar que, apesar de Plataforma ter apresentado o menor peso entre as dimensões (conforme Tabela 2), ela possuiu o melhor desempenho.

Sob a ótica do MáxCIS $\mathrm{S}$, a indústria tem maior potencial de crescimento nas dimensões: Oferta, Clientes, Soluções e Cadeia de Fornecimento. É necessário que os atores envolvidos analisem essas 
dimensões e estabeleçam ações e diretrizes estratégicas para alavancar a competitividade do setor e desenvolver a inovação. Entre os vetores apresentados para o MáxCIS $\mathrm{S}_{\mathrm{S}}$, Plataforma e Ambiência Inovadora apresentam o menor indicador de crescimento, no primeiro caso a dimensão já possuem um desempenho superior $\left(\mathrm{MedCIS}_{\mathrm{S}}\right)$, no segundo caso, a dimensão é tida como incipiente no setor.

Os resultados obtidos para o comércio estão descritos na Tabela 4. Plataforma, mais uma vez é o benchmark do setor, onde as dimensões que apresentam o maior desempenho em ordem decrescente são: Plataforma, Marca, Oferta, Relacionamento, Clientes e Organização. Entre as empresas concorrentes dessa atividade econômica, existe potencial de crescimento nas dimensões: Agregação de valor e Rede (com potencial de crescimento máximo entre as demais dimensões), além de Cadeia de Fornecimento, Soluções e Presença.

Tabela 4 - Característicos do setor de comércio no momento R2

\begin{tabular}{c|c|c|c|c|c}
\hline Dimensões & Média & Máximo & Mínimo & MedCIS & MáxCIS \\
\hline OFERTA & 3,707 & 5,000 & 1,000 & 3,888 & 1,293 \\
\hline PLATAFORMA & 4,767 & 5,000 & 3,000 & 5,000 & 0,233 \\
\hline MARCA & 3,933 & 5,000 & 3,000 & 4,126 & 1,067 \\
\hline CLIENTES & 3,117 & 4,300 & 1,700 & 3,269 & 1,376 \\
\hline SOLUÇÕES & 2,567 & 5,000 & 1,000 & 2,692 & 2,433 \\
\hline RELACIONAMENTO & 3,367 & 5,000 & 1,000 & 3,531 & 1,633 \\
\hline AGREGAÇÃO DE VALOR & 2,333 & 5,000 & 1,000 & 2,448 & 2,667 \\
\hline PROCESSOS & 2,120 & 3,300 & 1,300 & 2,224 & 1,788 \\
\hline ORGANIZAÇÃO & 2,900 & 4,000 & 1,500 & 3,042 & 1,375 \\
\hline CAD. FORNECIMENTO & 2,533 & 5,000 & 1,000 & 2,657 & 2,467 \\
\hline PRESENÇA & 1,600 & 3,000 & 1,000 & 1,678 & 2,333 \\
\hline REDE & 2,333 & 5,000 & 1,000 & 2,448 & 2,667 \\
\hline AMBIÊNCIA INOVADORA & 2,403 & 3,300 & 1,500 & 2,521 & 1,359 \\
\hline
\end{tabular}

Fonte: Dados da Pesquisa de Campo (2015).

Na Tabela 5 são apresentados os característicos para o setor de serviços. Como consequência da maior elasticidade entre as dimensões desse setor, ele possui várias dimensões que um bom desempenho (como consequência possui o maior GIS) em ordem decrescente tem-se: Rede (benchmark interno), Marca, Relacionamento, Soluções, Clientes, Plataforma, Organização e Oferta.

Nessas condições, os atores devem investir na agregação de valor prioritariamente e na cadeia de fornecimento para evitar estagnação da inovação nessa atividade econômica. Essas duas dimensões anteriormente analisadas mostram que se a pequena empresa possuir um conjunto logístico estruturado na entrega de um bem/serviço superior e captar o valor de seus bens oferecidos junto aos clientes promoverá a potencialização da inovação e poderá reforçar as demais dimensões que já possuem um bom desempenho. 
Tabela 5 - Característicos do setor de serviços no momento R2

\begin{tabular}{c|c|c|c|c|c}
\hline Dimensões & Média & Máximo & Mínimo & MedCIS & MáxCIS $_{\mathbf{s}}$ \\
\hline OFERTA & 2,767 & 5,000 & 1,000 & 3,05 & 2,233 \\
\hline PLATAFORMA & 3,733 & 5,000 & 1,000 & 4,12 & 1,267 \\
\hline MARCA & 4,000 & 5,000 & 2,000 & 4,41 & 1,000 \\
\hline CLIENTES & 3,747 & 5,000 & 1,700 & 4,13 & 1,253 \\
\hline SOLUÇÕES & 3,900 & 5,000 & 1,000 & 4,30 & 1,100 \\
\hline RELACIONAMENTO & 3,967 & 5,000 & 2,000 & 4,38 & 1,033 \\
\hline AGREGAÇÃO DE VALOR & 2,133 & 5,000 & 1,000 & 2,35 & 2,867 \\
\hline PROCESSOS & 2,620 & 4,300 & 1,300 & 2,89 & 1,953 \\
\hline ORGANIZAÇÃO & 3,583 & 5,000 & 2,000 & 3,95 & 1,417 \\
\hline CAD. FORNECIMENTO & 2,333 & 5,000 & 1,000 & 2,57 & 2,667 \\
\hline PRESENÇA & 2,067 & 5,000 & 1,000 & 2,28 & 2,933 \\
\hline REDE & 4,533 & 5,000 & 1,000 & 5,00 & 0,467 \\
\hline AMBIÊNCIA INOVADORA & 2,483 & 4,500 & 1,500 & 2,74 & 2,241 \\
\hline
\end{tabular}

Fonte: Dados da Pesquisa de Campo (2015).

Comparando os resultados obtidos pelos setores estudados, os dados revelam que, dada a escassez de recursos, investimentos devem priorizar o desenvolvimento da cadeia de fornecimento na indústria. Já no setor de comércio, devem-se priorizar ações para estimular a agregação de valor não só na entrega dos bem ao cliente, mas também na cadeia de fornecimento. Enquanto que possuir vários pontos de atendimento, ou seja, alta capilaridade deve ser prioridades para o oferecimento de serviços, dada à natureza de suas atividades e a conveniência para os clientes.

Estes resultados corroboram os estudos de Oliveira et. al (2014) e Oliveira, Cavalcanti e Paiva Jr. (2013), no qual constataram que o setor comercial possui maior vocação para a inovação frente aos segmentos industriais como gesso, eletrometalmecânica, móveis, confecções e de plástico.

\section{Gráfico 2 - Comparação entre os MedCISs no momento R2 para os 3 setores econômicos estudados}




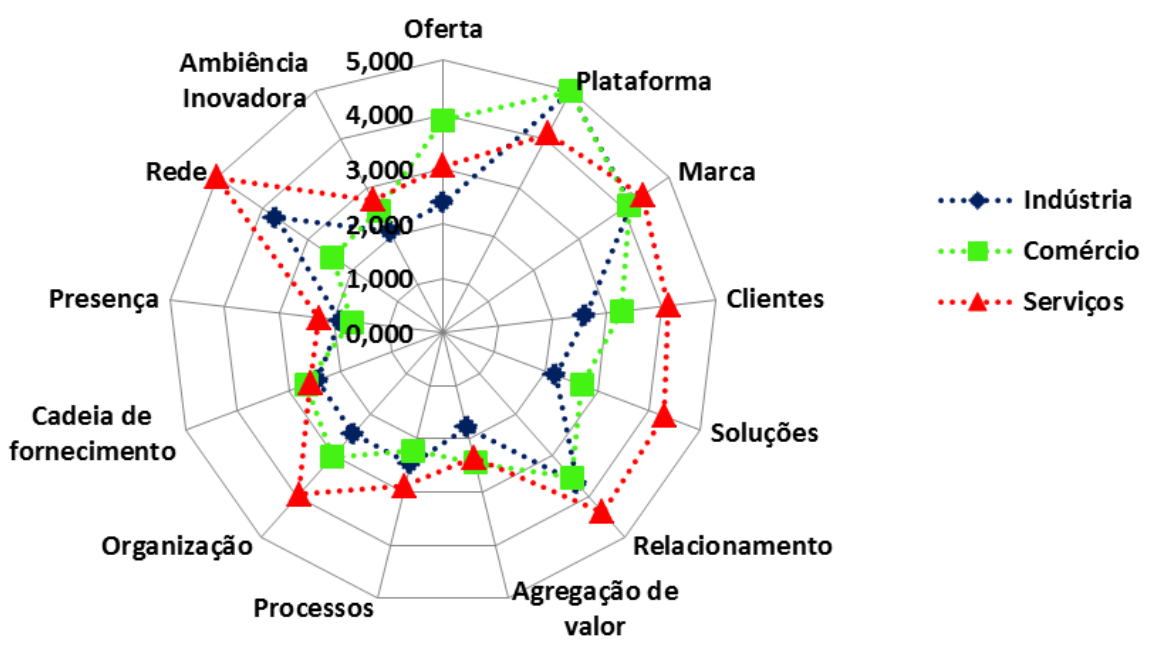

Fonte: Dados da Pesquisa de Campo (2014).

Realizando um exame visual (Gráfico 2) sobre as dimensões que apresentaram os melhores desempenhos, sob a perspectiva do MedCIS $\mathrm{S}$, o setor de serviços, em R2, possui uma grande maioria de dimensões com bom desempenho quando comparado com outros setores. Vale destacar que a dimensão ambiência inovadora, bastante valorizada na formação do GI tradicional, não apresentou nenhum desempenho superior entre os setores e não se apresenta como uma dimensão potencial de ser envolvida, segundo as análises realizadas.

\section{CONSIDERAÇÕES FINAIS}

Considera-se que o objetivo central do presente estudo foi cumprido, uma vez que os resultados obtidos permitiram uma avalição do grau de inovação (GI), do grau de inovação setorial (GIS) e do característico de inovação setorial (CIS) das EPPs pertencentes aos setores de indústria, comércio e serviços do estado de Pernambuco, sob uma perspectiva de análise intrassetorial contínua e intersetorial.

A aplicação do radar da inovação, sob a égide do GI, permitiu verificar que, no momento R2, o setor de serviços exibiu um avanço de $35 \%$, entre os períodos R0-R2, sendo o maior, seguido pelos setores de comércio $(26,3 \%)$ e indústria $(16,4 \%)$. Por sua vez, o setor comércio apresentou evolução do GI em todas as dimensões, enquanto o setor industrial obteve acréscimos em apenas seis dimensões do radar. Especificamente, a dimensão Plataforma se destacou nos setores de indústria e comércio, enquanto, Rede obteve destaque no setor de serviços. 
Com a aplicação do GIS foram identificadas as dimensões da inovação que mais contribuem para realizar e estruturar práticas inovadoras para o setor. Relacionamento, Clientes e Marca estiveram presentes como indutoras de peso no GIS nos três setores analisados, enquanto Plataforma e Rede estiveram presentes em dois dos três setores, como dimensões de peso. Além disso, tanto no GI quanto no GIS o setor de serviços apresentou o maior grau de inovação, seguido do setor de comércio e indústria.

Sob o parâmetro do MedCIS $_{\mathrm{s}}$, o setor de serviços apresentou a maioria das dimensões com forte desempenho com relação aos demais. A Plataforma foi considerada um ponto em que os setores comercial e industrial apresentaram excelente desempenho, como identificado pelo radar da inovação, implicando que as pequenas empresas de Pernambuco nesses segmentos possuem um conjunto de componentes e métodos estruturados para atender os seus clientes, o que de certo modo, contribui para o desenvolvimento de outras dimensões.

Já o MáxCIS s revelou quais dimensões apresentaram maior potencial para crescimento entre as empresas do mesmo setor. Como ponto crítico, foi observado que a dimensão Ambiência inovadora não mostrou notoriedade nas análises realizadas, revelando ainda pouca importância à propagação da cultura de inovação e a promoção de inovação como fonte e manutenção de vantagem competitiva. Isso se mostra como um entrave para o desenvolvimento das empresas, pois a ação inovadora do empresário engendra o progresso técnico que dinamiza o capitalismo. Nesse sentido, a inovação é a mola propulsora no estabelecimento do nível de competitividade no mercado, na produtividade das empresas e no progresso técnico-científico (Schumpeter, 1985).

Este estudo possibilitou a identificação das dimensões da inovação organizacional de maior impacto para cada setor econômico, assim como seu desempenho atual e potencial. Infere-se que políticas públicas podem ser elaboradas para promover o desenvolvimento dos pequenos empreendimentos do setor, que já contribuem de forma significativa no desenvolvimento da economia pernambucana. Por este motivo, consultorias, instutorias, cursos, capacitações podem ser desenvolvidas para atender as necessidades das EPPs, dada a sua realidade local e suas potencialidades.

Para estudos futuros, sugere-se o mapeamento de políticas públicas conforme as necessidades identificadas pelo radar da inovação e as ferramentas GIS e CIS para alavancar o desenvolvimento das pequenas empresas pernambucanas.

\section{REFERÊNCIAS}


Andreassi, T. (2007). Gestão da inovação metodológica. São Paulo: Thomson Learning.

Armbruster, H. et al. (2008). Organizational innovation: The challenge of measuring non-technical innovation in large-scale surveys. Technovation, n. 28, p. 644-657.

Bachmann, D. L.; Destefani, J. H. (2008). Metodologia para estimar o grau das inovações nas MPE. Curitiba.

BRASIL. Lei $n^{\circ} 10.973$, de 2 de dezembro de 2004. Dispõe sobre incentivos à inovação e à pesquisa científica e tecnológica no ambiente produtivo e dá outras providências. DOU, Brasília, n. 232, 3 dez. 2004.

Carayannis, E. G.; Provance, M. (2008). Measuring firm innovativeness: towards a composite innovation index built on firm innovative posture, propensity and performance attributes. International Journal of Innovation and Regional Development, 1(1), p. 90-107.

Creswell, J. W. (2010). Projeto de pesquisa: métodos qualitativo, quantitativo e misto. $3^{\mathrm{a}}$ ed. Porto Alegre: Artmed/Bookman, p. 296.

Drucker, P. F. (2002). Inovação e espírito empreendedor. Tradução de Carlos Malferrari. São Paulo: Pioneira Thomson.

Hronec, S. M. (1994). Sinais vitais: usando medidas de desempenho da qualidade, tempo e custo para traçar a rota para o futuro de sua empresa. São Paulo: Makron Books, p. 240.

Julien, P-A. (2010). Empreendedorismo regional e economia do conhecimento. São Paulo: Saraiva.

Lara, F. F. \& Guimarães, M. R. N. (2013). Inovação organizacional: estudos de caso comparativos entre duas empresas de pequeno porte e duas empresas subsidiárias do setor metal-mecânico da região de Sorocaba. GEPROS. Gestão da Produção, Operações e Sistemas, Bauru, Ano 8, nº 1, p. 95-107.

Moreira, D. \& Queiroz, A. C. (Org.). (2007). Inovação organizacional e tecnológica. São Paulo: Thomson.

Oliveira, M. R. G. et al. (2011). Grau de Inovação Setorial - uma abordagem a partir do radar de inovação. In: Encontro Nacional de Engenharia de Produção, ENEGEP, 31. Belo Horizonte. Anais do ... Belo Horizonte: ABEPRO, 2011a, p. 1-13.

Oliveira, M. R. G. et al. (2011). Grau de Inovação de micro e pequenas empresas pernambucanas: um estudo comparativo. In: Simpósio de Engenharia de Produção - SIMPEP, 18. Bauru, SP. Anais do ... São Paulo: UNESP, 2011b, p. 1-14.

Oliveira, M. R. G. et al. (2014). Mensurando a inovação por meio do grau de inovação setorial e do característico setorial de inovação. Revista de Administração e Inovação - RAI. São Paulo, SP, v. 11, n.1, p. 113-137.

Oliveira, M. R. G., Cavalcanti, A. M., \& Paiva Jr, F. G. (2013). Mensurando a inovação por meio do grau de inovação setorial e do característico setorial de inovação. In: Simpósio de Administração da Produção, Logística e Operações Internacionais - SIMPOI, 16. São Paulo, SP. Anais do ... São Paulo: FGV/EAESP, p. 1-16.

Revista de Administração e Inovação, São Paulo, v. 12, n.4 p. 140-161, out./dez. 2015. 
Organização para Cooperação Econômica e Desenvolvimento (OCDE). (2006). Manual de Oslo: diretrizes para coleta e interpretação de dados sobre inovação. 3. ed. Brasília: FINEP.

Paredes, B. J. B., Santana, G. A., \& Fell, A. F. A. (2014). Um estudo de aplicação do radar da inovação: o grau de inovação organizacional em uma empresa de pequeno porte do setor metalmecânico. Navus revista de gestão e tecnologia, Florianópolis, 4(1), p. 76-88.

Paredes, B. J. B., Valente, B., \& Santana, G. A. (2013). A marca como diferencial de inovação: um estudo em empresas de pequeno porte do segmento metalmecânico em recife/pe. In: simpósio de Engenharia de Produção, 20, SIMPEP, Bauru. Anais do XX SIMPEP, Bauru, SIMPEP.

Rocha, M. J. F. et al. (2011). Análise da influência da estrutura organizacional no processo de inovação nas micro e pequenas empresas do setor da indústria moveleira de pernambuco. In: simpósio de Engenharia de Produção, 18, SIMPEP, Bauru. Anais do XX SIMPEP, Bauru, SIMPEP.

Santana, G. A., Paredes, B. J. B., \& Valente, B. (2013). Inovação em empresas organizadoras de eventos: análise da dimensão relacionamento do radar da inovação. In: simpósio de Engenharia de Produção, 20, SIMPEP, Bauru. Anais do XX SIMPEP, Bauru, SIMPEP.

Sawhney, M., \& Chen, J. (2010). Defining and Measuring Business Innovation: The Innovation Radar. IN: Proceedings of the American Marketing Association Conference, Boston.

Sawhney. M., Wolcott, R., \& Arroniz, I. (2006). As 12 dimensões da Inovação. MIT Sloan Management Review, Cambridge, p. 75-81.

Schumpeter, J. A. (1985). Teoria do desenvolvimento econômico: uma investigação sobre lucros, capital, credito, juro e o ciclo econômico. 2. ed. São Paulo: Nova Cultural.

Schumpeter, J. A. (1988). A teoria do desenvolvimento econômico. São Paulo: Nova Cultural.

Silva Néto, A. T., \& Teixeira, R. M. (2011). Mensuração do grau de inovação de micro e pequenas empresas: estudo em empresas da cadeia têxtil-confecção em Sergipe. Revista de Administração $e$ Inovação, São Paulo, 8(3), p. 205-229, jul ./set.

Silva, M. E. (2013). Inovação Organizacional para o Consumo Sustentável: a inserção de novas práticas e tecnologias nos negócios do Walmart Brasil. Reunir - Revista de Administração, Ciências Contábeis e Sustentabilidade, 3, p. 66-89.

Tidd, J., Bessant, J. \& Pavitt, K. (2008). Gestão da Inovação. Porto Alegre: Bookman.

Ukko, J., \& Saunila, M. (2013). The role of reflection in facilitating and assessing innovativeness. Journal of Technology Management \& Innovation [online], 8(4), p. 170-176. 


\title{
AN ANALYSIS INTRASECTORAL INTERSECTORAL AND SMALL BUSINESS INNOVATION DEGREE OF PORTE PERNAMBUCO STATE
}

\begin{abstract}
The development of innovation in companies has as its foundation the effort of continuity in the generation of performance indicators. However, measurements and evaluations of companies are often guided by objectives and short-term effects, making it impossible to continue the effort in generating parameters. For the process of gradual improvement of organizational performance, assessments of the degree of innovation must be continuous. Given this, this study evaluates the Degree of Innovation, the Degree of Innovation Sector and the Characteristic of Sectoral Innovation of Small Businesses belonging to the Industry, Trade and Services sectors of the state of Pernambuco, from a perspective of continuous intra-sectoral and inter-sectoral analysis. To achieve this objective, the diagnostic Innovation Radar was applied on 90 EPPs in three different times. The results showed that the service sector has shown greater progress in innovation compared to trade and industry. Once the trade sector showed an increase of GI in all dimensions, while the industrial sector had accrued in just six dimensions of the radar. Through GIS, we found that the relationship, Brand and Customer dimensions stood out as inducing weight in the three sectors analyzed.
\end{abstract}

Key words: Innovation; Radar Innovation; Micro and Small businesse; Degree of sectoral innovation.

Data do recebimento do artigo: 07/01/2015

Data do aceite de publicação: 06/09/2015

Revista de Administração e Inovação, São Paulo, v. 12, n.4 p. 140-161, out./dez. 2015. 